\title{
Bewertung des Kosten-Nutzen-Verhältnisses einer Demontagelinie am Standort des Europäischen Transport- und Logistikzentrums Preschen
}

\author{
Kirsten Wechsel
}

\section{Einführung}

Im Rahmen des Projektes zur „Entwicklung eines Demontagesystems für Waschmaschinen innerhalb eines Wertstoffkreislaufes" (vgl. Artikel Hentschel/Kindler) wurde durch eine Diplomarbeit das Kosten-Nutzen-Verhältnis dieser Demontagelinie am Standort des Europäischen Transport- und Logistikzentrums Preschen untersucht und bewertet. Diese Untersuchung wird die Grundlage für die weitere Planung des Projektes bilden. Die Ergebnisse dieser Arbeit werden im Folgenden kurz dargestellt.

\section{2. Ökonomische Ausgangsbasis}

Der eigentlichen Kosten-Nutzen-Betrachtung geht die Erarbeitung der logistischen Rahmenbedingungen voraus, die eine wesentliche Grundlage der Kosten- und Erlösermittlung bilden. Hierbei ist es notwendig einige Arbeitsannahmen zu treffen. Eine der wichtigsten Arbeitsannahmen ist die Festlegung der Menge an zu demontierenden Geräten. Ausgangspunkt für die Berechnung des Annahmewertes ist die Kapazität der Anlage. Die maximal zu demontierende Menge je Jahr ist dabei mit der Menge gleichzusetzen, die in dieser Anlage in einem Jahr demontiert werden kann. Wird bei $250 \mathrm{Ar}$ beitstagen eine Systemverfügbarkeit von $98 \%$ zugrunde gelegt, so ist von 245 Arbeitstagen je jahr auszugehen. Bei einer täglichen Arbeitszeit von 8 Stunden und ein-
Bezeichnung

Lagerkosten gesamt

Kalkulatorische Abschreibungen

Kalkulatorische Zinsen

Kosten für Fremdkapital

Personalkosten

Wartungs- und Instandhaltungskosten

Raumkosten

Versicherungskosten

Energiekosten

Kosten für die Entsorgung der Reststoffe

Gesamtkosten

Erlöse für die Annahme von Altgeräten

Erlöse fuir den Verkauf an Sekundär-

rohstoffen

Kosten in DM/a

$3.946,00$

$109.318,00$

$17.741,00$

$8.266,00$

$176.400,00$

$3.600,00$

$9.240,00$

4.613,00

$5.994,00$

$25.210,00$

$364.328,00$

$100.800,00$

$170.005,00$

Gesamterlöse

$270.805,00$
Tabelle 1: Zusammenfassung der Kosten und Erlöse schichtigem Betrieb ergibt sich eine Gesamtarbeitszeit von 1960 Stunden. Kann in dieser Anlage im Durchschnitt alle 700 Sekunden eine Waschmaschine komplett zerlegt werden, so können im Jahr ca. 10.080 Geräte demontiert werden. Auf Grundlage dieser Ausgangsgröße konnten die in den folgenden Tabellen zusammengefaßten Kosten und Erlöse ermittelt werden.

\section{Bewertung des Kosten-Nutzen- Verhältnisses}

Der Gegenüberstellung der Kosten und des Nutzen bzw. der Erlöse gehen im Folgendem einige grundlegende Bemerkungen voraus. Für die Beurteilung des KostenNutzen-Verhältnisses ist es wichtig zu wissen, daß es sich sowohl auf der Kosten- als auch auf der Erlösseite um Prognosen handelt, die sich teilweise auf mehr oder weniger realistische Annahmen stïtzen. So bauen sich beispielsweise die gesamten Berechnungen der Erlöse und teilweise auch der Kosten auf die Mengenannahme von 10.080 Altgeräten je Jahr auf. Es ist derzeit nicht gesichert, daß das Mengenaufkommen in der Region auch der Kapazität der Anlage entspricht, bzw. ob die rechnerische Kapazität der Anlage sich in der Praxis bestätigt. Weitergehende Untersuchungen zum Aufkommen in anderen Regionen sind deshalb erforderlich.

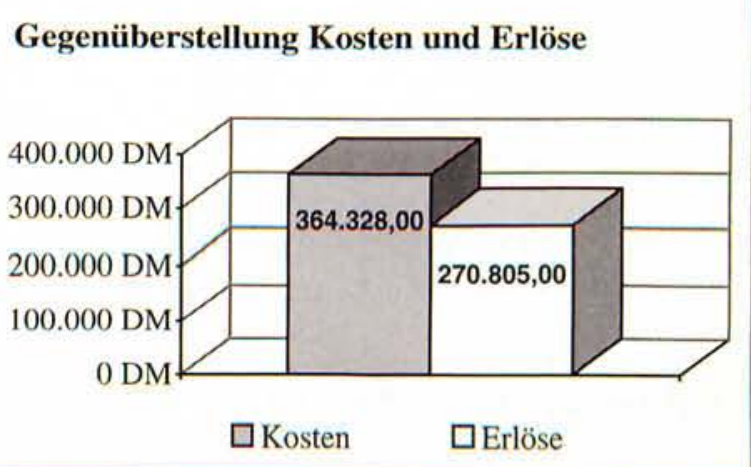

Abb. 1: Gegenüberstellung Kosten und Erlöse

Auf Grundlage der Projektergebnisse zur Planung des Demontagesystem wurde innerhalb der Diplomarbeit ein Kostenaufwand in Höhe von 364.328,00 DM errechnet. Die Ergebnisse zeigen, daß die Gesamtkosten deutlich über den zu erwartenden Erlösen liegen. Wird davon ausgegangen, daß im Jahr 10.080 Geräte demontiert werden, so ist mit einem Verlust von 93.523,00 DM 
jährlich zu rechnen. Es ist nun zu betrachten, inwieweit die Erlöse die variablen Kosten decken bzw. bei welcher Menge an demontierten Altgeräten die Gesamtkosten gedeckt werden könnten.

\section{Break-Even-Analyse}

Die Break-Even-Analyse wird auch als Gewinnschwellen-, Deckungspunkt-Analyse bezeichnet. Mittels der Break-Even-Analyse läßt sich der Zusammenhang von Erlösen und Kosten sowie von Gewinnen und Verlusten in Hinsicht auf verschiedene Beschäftigungsgrade darstellen. Für die in dieser Arbeit betrachtete Demontagelösung wurde allen Kosten- und Erlösberechnungen eine maximale Kapazitätsauslastung bei einschichtigem Betrieb zugrunde gelegt. Ausgehend von diesem Aspekt ist es nun von besonderem Interesse festzustellen, ab welcher Kapazitätsauslastung mit der Anlage Gewinne erwirtschaftet werden können. In Hinsicht auf die Fragestellung nach der Anzahl der Geräte, die mindestens demontiert werden müssen, um Gewinn erwirtschaften zu können, ist die Menge, bei der weder ein Betriebsgewinn noch -verlust entsteht, von großer Bedeutung.

Dieser Punkt wird als Break-Even-Point bezeichnet und stellt den „Gewinn- bzw. Verlustschwellenpunkt“ dar.

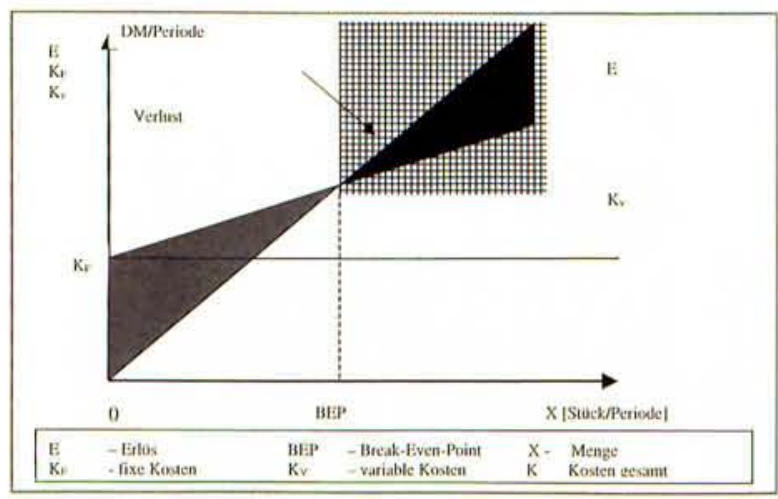

Abb. 2: Break-Even-Analyse mit Erlös-Kosten-Modell

Der Break-Even-Point berechnet sich, indem die Erlöse mit den Kosten gleichgesetzt werden. $(\mathrm{E}=\mathrm{K}$ bzw. e $\mathrm{x}=$ $\mathrm{K}_{\mathrm{v}} \mathrm{x}+\mathrm{K}_{\mathrm{F}}$ ) Nach Umstellung ergibt sich für die Berechnung folgende Formel:

$$
\mathrm{X}_{\mathrm{BEP}}=\frac{\mathrm{K}_{\mathrm{F}}}{\mathrm{e}-\mathrm{k}_{\mathrm{v}}}
$$

Zur Berechnung der Gewinnschwelle müssen die durch die Demontageanlage verursachten Kosten zunächst in fixe und variable Kosten unterteilt werden.

Werden die variablen Kosten insgesamt durch die Anzahl der demontierten Geräte dividiert, so ergeben sich die variablen Kosten je demontiertes Altgerät. Bei einer Menge von 10.080 Altgeräten entspricht dies variablen Kosten von 20,60 DM je Gerät.

Bei einem Gesamterlös von 270.805,00 DM je Jahr be-

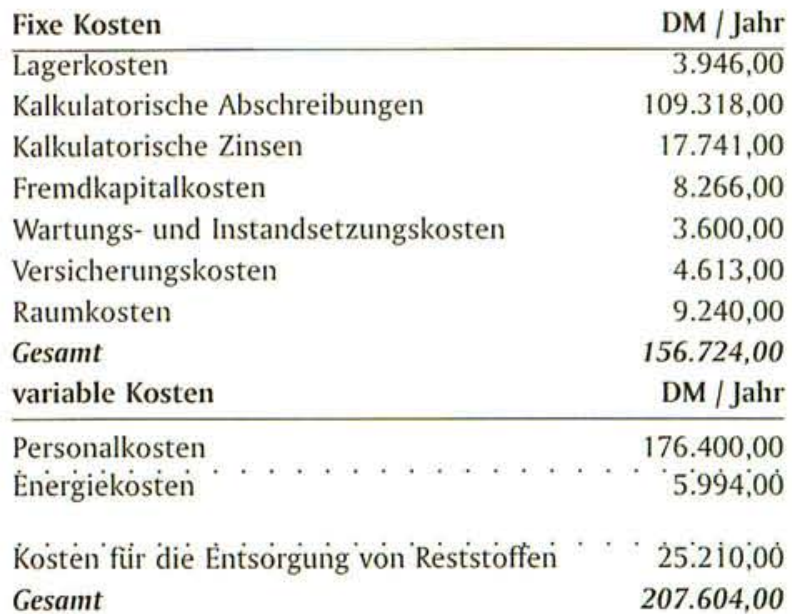

Tabelle 2: Einteilung der Kosten in fixe und variable Kosten

tragen die Erlöse je Gerät 26,90 DM. Die Menge der zu demontierenden Altgeräte, bei der die Kosten gleich den Erlöse sind, berechnet sich folgendermaßen:

$$
\begin{array}{r}
156.724,00 \\
X_{B E P}=\quad \begin{array}{r}
26,90-20,60
\end{array}
\end{array}
$$

wobei der Break-Even-Point bei einer Menge von 24.877 Geräten liegt. D.h., bei der Demontage von 24.877 Altgeräten erwirtschaftet die Anlage weder einen Verlust noch einen Gewinn.

\section{Schlußbetrachtungen}

Das Ergebnis der bisherigen Berechnungen läßt den Schluß zu, daß die Demontageanlage für Waschmaschinen bei einem einschichtigen Betrieb nicht wirtschaftlich ist. Wenn der Break-Even-Point bei einer Menge von 24.877 Geräten liegt, so ist dies bei einer Kapazität von 10.080 Geräten je Schicht nur bei einem dreischichtigen Betrieb zu erreichen. Wird ein dreischichtiger Betrieb angenommen, so erweitert sich die Kapazität der Demontageanlage auf 30.240 Geräte je Jahr. Bei einem Erlös von 26.90 DM je demontiertes Altgerät ermittelt sich ein Gesamterlös von 813.456,00 DM je Jahr. Die jährlichen Kosten setzten sich aus den fixen Kosten von 156.724,00 DM und den variablen Kosten zusammen. Belaufen sich die variablen Kosten auf 20.60 DM je demontiertes Alt-

\section{Hochrechnung der Kosten und Erlöse}

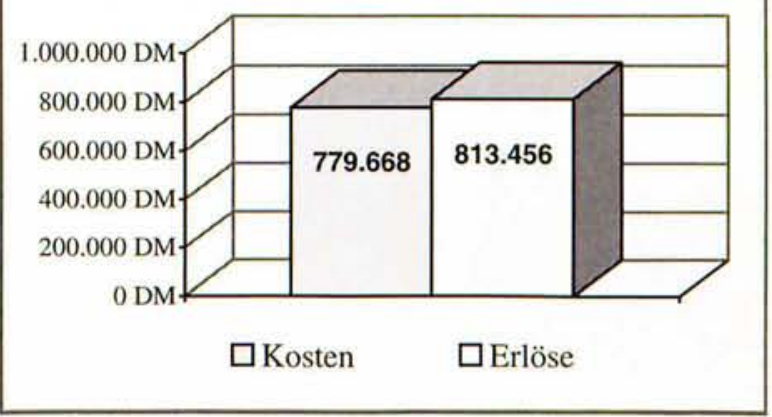

Abb. 3: Hochrechnung der Kosten und Erlöse bei Dreischichtbetrieb 
gerät, so sind dies 622.944,00 DM im Jahr. Demzufolge stehen den jährlichen Erlösen von 813.456,00 DM Gesamtkosten in Höhe von 779.668,00 DM gegenüber.

Die bedeutet, daß die Anlage bei dreischichtigem Betrieb einen Gewinn von 33.788,00 DM erwirtschaften könnte. Für die weitere Planung eines Demontagesystems im Euro Trans-Logistics-Centre (E.T.L.C.) konnte somit eine erste ökonomische Bewertung zur Sicherung einer gewinnorientierten Demontage vorgelegt werden.

Im Zusammenhang mit der Erweiterung des Demontagesystems für andere zu demontierende Altprodukte zeichnet sich somit eine positive Tendenz zum Betreiben eines derartigen Systems ab.

\section{Verfasserin}

\section{Dipl.-Betriebswirtin (FH) Kirsten Wechsel}

Absolventin der Technischen Fachhochschule Wildau Schelchem GmbH Im Gewerbepark 6, 15711 Zeesen

Tel. (0 33 75) 903830 